\title{
Tasisulam Sodium
}

National Cancer Institute

\section{Source}

National Cancer Institute. Tasisulam Sodium. NCI Thesaurus. Code C88304.

The sodium salt of an acyl-sulfonamide with potential antineoplastic activity. Selectively toxic towards tumor cells, tasisulam appears to induce tumor cell apoptosis by a mitochondrial-targeted mechanism involving the loss of mitochondrial membrane potential and induction of reactive oxygen species (ROS). In combination with an angiogenesis inhibitor, this agent may exhibit synergistic antiangiogenic activity. 